\title{
EL PROBLEMA DE LA GRADACIÓN ONTOLÓGICA DEL ESSE IMMATERIALE EN TOMÁS DE AQUINO
}

\author{
Fernando Gabriel Hernández \\ Universidad de Buenos Aires \\ Consejo Nacional de Investigaciones Cientificas y Técnicas \\ bttp://dx.doi.org/10.15304/ag.38.2.5489
}

\begin{abstract}
Resumen
Aristóteles afirma en el De Anima que percibir consiste en recibir una forma sensible sin materia. Cuando Tomás de Aquino comenta este pasaje precisa la definición del Estagirita y sostiene que esa forma sensible posee una existencia intencional (esse intentionale) en el sujeto cognoscitivo. De esta manera, denomina inmutación espiritual (immutatio spiritualis) al proceso por el cual un ente recibe una forma sensible que posee una existencia intencional. Los problemas respecto a la inmutación espiritual surgen cuando se estudia en profundidad su status ontológico, puesto que en varias de sus obras el Aquinate declara que se trata de una inmutación inmaterial, pero en muchas afirma que es una inmutación material. El objetivo de este artículo es resolver esta aparente contradicción y demostrar que para Tomás de Aquino existe una gradación ontológica.

Palabras clave: Tomás de Aquino, ser intencional, percepción, inmutación espiritual.
\end{abstract}

\begin{abstract}
In the De Anima Aristotle affirms that to perceive is to receive a sensible form without matter. When Thomas Aquinas comments this passage specifies the definition of the Stagirite and holds that this sensible form has an intentional being (esse intentionale) in the cognitive subject. In this way, he calls spiritual change (immutatio spiritualis) to the process by which a being receives a sensible form that has an intentional existence. The problems about the spiritual change arise when its ontological state is studied. Aquinas declares in several works that it is an immaterial immutation, but in many other works he affirms that it is a material
\end{abstract}

Recibido: 16/09/2018. Aceptado: 24/01/2019. 
immutation. The aim of this paper is to solve this apparent contradiction, this is possible if it is demonstrated that for Thomas Aquinas there is an ontological gradation.

Keywords: Thomas Aquinas, intentional being, perception, spiritual change.

\section{Introducción}

Para Tomás de Aquino un ente es cognoscitivo si posee formas de otros entes, ${ }^{1}$ las cuales tienen una existencia intencional en el sujeto cognoscente. ${ }^{2}$ Es innegable que tales formas intencionales tienen una existencia inmaterial en el intelecto; sin embargo, el Aquinate es ambiguo con respecto al status ontológico de las formas que se encuentran en los sentidos, ya que algunas veces afirma que las mismas se reciben materialmente ${ }^{3}$ y otras veces que se reciben inmaterialmente en los órganos sensorios. ${ }^{4} \mathrm{El}$ objetivo del artículo es estudiar el status ontológico de las formas que se encuentran en el sentido a partir del estudio de la inmutación espiritual. El vínculo entre la immutatio spiritualis y las formas que tienen un esse intentionale es tan estrecho que el conocimiento del status ontológico de una revelará la naturaleza de la otra.

A lo largo del artículo se intentará mostrar que la inmutación espiritual no puede ser entendida como una alteración psíquica o del alma, como se pretendió clásicamente, ni como una alteración material, como suelen interpretar algunos autores contemporáneos. Estas lecturas fallan al pensar la realidad de manera dicotómica, esto es, pensar que algo es o bien material, o bien inmaterial. Por el contrario, para Tomás de Aquino la realidad se manifiesta en una gradación, es por eso que la forma sensible puede tener un estado intermedio entre la materialidad y la inmaterialidad en la inmutación espiritual. A su vez, se mostrará que el concepto spiritus, contenido en el término immutatio spiritualis y en la denominación de la existencia intencional como esse intentionale et spirituale, ${ }^{5}$ es el más apropiado para indicar este estado intermedio.

Para lograr estos objetivos se dividirá el artículo tres capítulos:

1) En el primer capítulo se expondrá la teoría de la percepción de Tomás de Aquino y la distinción que establece entre una inmutación natural y una inmutación espiritual. También se presentarán las posiciones que entienden

${ }^{1}$ Cfr. Tomás de Aquino, S. Th. I, q.14, a.1, co.

${ }^{2} \mathrm{C} f r$. Tomás de Aquino, In De Anima II lectio 24, 551.

${ }^{3} \mathrm{C} f r$. Ibid, lectio12, 377.

${ }^{4} \mathrm{C} f r$. Tomás de Aquino, Compendium theologiae, cap. 52.

${ }^{5}$ Cfr. Tomás de Aquino, In De Anima II lectio 24, 553. 
la inmutación espiritual como un cambio material ${ }^{6}$ y a quienes interpretan que tal inmutación es algo meramente psicológico e inmaterial. ${ }^{7}$

2) En el segundo capítulo se presentará la postura de Jörg Tellkamp ${ }^{8}$ para quien hay evidencias textuales que permiten sostener las dos posiciones mencionadas. Esto es, hay pasajes en los que Tomás afirma que la inmutación espiritual y, por tanto, las formas sensibles son inmateriales pero también existen lugares donde sostiene que las mismas son materiales. Para este autor dicha contradicción se refleja en el concepto de spiritus que utiliza el Aquinate, puesto que lo recibe de dos fuentes opuesta: a) La teoría estoica del pneuma que hace referencia a un soplo puramente material y b) La tradición agustiniana y bíblica que donde se usa este concepto para tratar de seres inmateriales.

3) En el tercer capítulo se analizará el status ontológico de la forma sensible. Se mostrará que para Tomás de Aquino el pasaje que va desde los seres materiales a los seres inmateriales se presenta en una gradación. Los seres que poseen conocimiento sensible se encuentra en el medio de esta gradación y por ello es que la percepción consiste en una inmutación que no es ni material ni inmaterial, sino que es semi-inmaterial, esto es, está en un estado intermedio entre la materialidad y la inmaterialidad. Además, se señalará que el concepto de spiritus, lejos de manifestar una contradicción en la teoría de la percepción en Tomás, resulta ser el término más indicado para designar dicho estado intermedio.

\section{Inmutación espiritual: material o inmaterial}

Tomás de Aquino clasifica a los poderes sensitivos en dos categorías: los sentidos internos y los sentidos externos. Cuando se habla de sentidos de manera general se hace referencia a los sentidos externos, los cuales son cinco: vista, oído, tacto, olfato y gusto. Estos sentidos se distinguen entre sí no tanto por los órganos como por sus diversas funciones. Para el Aquinate un sentido es un poder pasivo cuya función es estar sometido a la acción

${ }^{6}$ Cfr. Cohen, S. "St. Thomas Aquinas on the Immaterial Reception of Sensible Forms", Philosophical Review 91 (1982),193-209; Pasnau R. Theories of cognition in the later Middle Ages, Cambridge, Cambridge University Press, 1997.

${ }^{7}$ Cfr. Hamlyn, D. Sensation and Perception, London, Routledge \& Kegan Paul, 1961; Burnyeat M. "Is an Aristotelian Philosophy of Mind Still Credible? (A Draft)", Essays on Aristotle's de Anima (1995) 15-26.

${ }^{8}$ Tellkamp J, “Espíritu e inmutación espiritual: desarrollos y problemas en Tomás de Aquino”, Tópicos: Revista de Filosofía, 15 (1998), 181-210. 
de los objetos externos. Los entes físicos son la fuente externa que produce un cambio interno en el sujeto cognoscitivo, diversificando los sentidos acuerdo a la diversidad de los objetos. La vista, por ejemplo, se diferencia del oído porque su objeto es el color y el del oído es el sonido y no porque sus órganos son los ojos y las orejas, respectivamente. ${ }^{9}$

En relación a la acción a la que están sometidos los sentidos, cabe señalar que Tomás de Aquino sigue las enseñanzas de Aristóteles. El Estagirita afirma en el De anima que la sensación, en sentido general, "tiene lugar cuando el sujeto es movido y padece una afección: parece tratarse, en efecto, de un cierto tipo de alteración". ${ }^{10}$ Es importante destacar que Aristóteles se ve obligado a utilizar la palabra "alteración" debido a que no contaba con otro término que exprese adecuadamente lo que sucede en la sensación y, por eso mismo, agrega la palabra "cierta", dando a entender que la sensación no debe ser entendida como una alteración tal y como es definida en la Física.

Tomás de Aquino rescata dicha distinción entre una alteración física y esta "cierta alteración" que se produce en la sensación y, por eso, introduce un nombre concreto para cada una de estas inmutaciones y habla de una inmutación natural y de una inmutación espiritual respectivamente. Así, cuando en la Suma Teológica trata sobre los sentidos externos señala esta distinción y remarca la importancia de la inmutación espiritual para el conocimiento sensible:

La inmutación natural se da cuando la forma de lo que es causa de la alteración es recibida en el objeto alterado según su propio ser natural (esse naturale). Ejemplo: El calor en lo calentado. La inmutación espiritual se da cuando la forma de lo que provoca la alteración es recibida en el objeto alterado según su ser espiritual (esse spirituale). Ejemplo: La forma del color en la pupila, la cual, no por eso queda coloreada. Para la operación del sentido se requiere una inmutación espiritual por la que se establezca en el órgano del sentido una representación intencional de la forma sensible. De no ser así, si sólo la alteración natural fuera necesaria para sentir, todos los cuerpos físicos, al ser alterados, sentirían. ${ }^{11}$

${ }^{9}$ Cfr. Kenny, A. Aquinas on Mind, New York, Routledge, 1993, p. 33.

${ }^{10}$ Aristóteles, De anima 416b 34-35.

${ }^{11}$ Tomás de Aquino, S.Th. I, q. 78, a. 3, co.: "Naturalis quidem, secundum quod forma immutantis recipitur in immutato secundum esse naturale, sicut calor in calefacto. Spiritualis autem, secundum quod forma immutantis recipitur in immutato secundum esse spirituale; ut forma coloris in pupilla, quae non fit per hoc colorata. Ad operationem autem sensus requiritur immutatio spiritualis, per quam intentio formae sensibilis fiat in organo sensus. Alioquin, si sola immutatio naturalis sufficeret ad sentiendum, omnia corpora naturalia sentirent dum alterantur." 
Es importante destacar dos cosas en este pasaje: 1) cuando un ente es inmutado espiritualmente, recibe una forma según un ser espiritual o intencional; y 2) la inmutación espiritual es presentada como una condición necesaria para el conocimiento sensible, incluso puede ser pensada como el criterio de demarcación entre los seres cognoscitivos y no cognoscitivos. Considero que la comprensión del término spiritus, que forma parte del concepto de inmutación espiritual, puede ayudar a comprender algunos aspectos de la teoría tomista de la percepción.

Hasta finales del siglo XX existió una interpretación clásica del conocimiento sensible en el pensamiento del Aquinate, David W. Hamlyn se nos presenta como un claro ejemplo de esta visión. En su libro titulado Sensation and Perception: A History of the Philosophy of Perception esboza esta interpretación y en ella postula una lectura particular de la alteración espiritual. Según sus palabras, para Tomás de Aquino la sensación consiste en dos cambios o alteraciones. La primera inmutación es un cambio físico que ocurre en el órgano sensorio y lo altera materialmente. Tomás caracteriza este cambio como la recepción natural de la forma sensible. Y la segunda inmutación, la immutatio spiritualis, consiste en un cambio espiritual o mental que se da únicamente en el alma, razón por la cual Tomás la denomina alteración espiritual:

[Aquino] ve la percepción sensible primariamente como una forma de cambio en la que el órgano sensorio es alterado. Pero esto no puede ser todo lo que esta envuelve, junto con el cambio físico va la recepción de una forma sensible sin materia. A este último Aquino lo toma no como siendo algo que ocurre en el órgano sensible, sino algo que sucede a la facultad del alma o la mente. Esto es, en sus palabras, un cambio espiritual. En esto difiere de Aristóteles, ya que, como indiqué anteriormente, es el órgano sensorio el que, según la visión de Aristóteles, recibe la forma sensible. Aquino observa que no puede decirse que el ojo recibe el color del objeto de la visión, y en consecuencia coloca un refinamiento a la doctrina de Aristóteles. De manera similar, trata la asimilación como una verdad sobre lo que ocurre en la mente en la percepción, no en el órgano sensorio. ${ }^{12}$

${ }^{12}$ Hamlyn, D. op. cit., p. 46: "[Aquinas] views sense-perception primarily as a form of change in which the sense-organ is altered. But this cannot be all that is involved, for along with the physical change there goes the reception of a sensible form without the matter. The latter Aquinas takes to be not something that happens to the sense-organ, but something that happens to the faculty of the soul or mind. It is, in his words, a spiritual change. In this he differs from Aristotle, since, as I indicated earlier, it is the sense-organ which, on Aristotle's view, receives the sensible form. Aquinas sees that the eye cannot be said to receive the colour of the object of vision, and in consequence he puts a refinement upon Aristotle's doctrine. In a similar way, he treats come assimilated as a truth about what happens to the mind in perception, not the sense-organ." 
Como mencionamos anteriormente, esta interpretación predominó entre los comentadores del Aquinate hasta convertirse en la lectura "canónica" de la distinción entre una inmutación espiritual y una inmutación natural. Para nuestro trabajo es importante remarcar que en ella se entiende que la inmutación espiritual se da en el alma y la inmutación natural en los órganos sensorios. La percepción ocurre cuando el órgano es inmutado naturalmente y el alma es inmutada espiritualmente. El caso de la visión sería algo extraordinario porque basta con la sola inmutación espiritual, como el mismo Tomás de Aquino afirma. ${ }^{13}$

Myles Burnyeat sostiene una lectura parecida a la de Hamlyn. En los debates que mantiene con Richard Sorabji sobre la percepción en Aristóteles, afirma que encontró en la teoría de la inmutación espiritual del Aquinate la apropiada interpretación de la doctrina del Estagirita. Para decirlo de manera rápida y sencilla, Burnyeat sostiene la percepción consiste pura y exclusivamente en la inmutación espiritual que menciona Tomás de Aquino.

Todas esas descripciones aparentemente físicas-los órganos llegando a ser como el objeto, su ser afectado, actualizado o alterado por las cualidades sensibles, su tomar la forma sensible sin la materia- todo esto se refiere a lo que Aquino llama una "inmutación espiritual", un tomar conciencia de alguna cualidad sensible en el entorno. ${ }^{14}$

Aunque las interpretaciones de Hamlyn y Burnyeat son diferentes, puesto que para el primero es necesario que el órgano sensorio sea inmutado naturalmente para que haya percepción mientras que el segundo niega tal necesidad, ambos coinciden en pensar a la inmutación espiritual como una modificación del alma o de la mente. La distinción entre la inmutación natural y la inmutación espiritual, para estos dos comentadores, reside en el hecho de que la primera consiste en un cambio material o físico y la segunda en un cambio mental o del espíritu. De ahí que Burnyeat considere que Tomás de Aquino denomina "inmutación espiritual" a la toma de conciencia de alguna cualidad sensible por parte del sujeto.

Sin embargo, esta lectura ha sido criticada por Sheldon Cohen, quien ha mostrado de manera convincente que tal explicación no es plausible por el hecho de que va en contra de numerosas evidencias textuales donde Tomás de Aquino sostiene que la inmutación espiritual se da en el órgano sensorio

${ }^{13}$ Cfr. Tomás de Aquino, In De Anima I, lectio 10, 189.

${ }^{14}$ Burnyeat, M. op. cit., p. 21: “All these physical-seeming descriptions-the organ's becoming like the object, its being affected, acted on, or altered by sensible qualities, its taking on sensible form without the matter-all these are referring to what Aquinas calls a 'spiritual' change, a becoming aware of some sensible quality in the environment." 
y no en el alma. Así cuando en la Suma Teológica trata sobre los sentidos externos señala que:

La inmutación espiritual se da cuando la forma de lo que provoca la alteración es recibida en el objeto alterado según su ser espiritual. Ejemplo: La forma del color en la pupila, la cual, no por eso queda coloreada. ${ }^{15}$

Al afirmar que la pupilla u oculo son inmutados espiritualmente, Tomás está estableciendo que esta inmutación se da en un órgano sensorio que tiene la particularidad de que una potencia del alma sensitiva vendría a ser como la forma de su materia. ${ }^{16}$ Es decir, hasta el momento parecería ser que la inmutación espiritual requiere que el objeto inmutado sea un ser animado. De esta manera seguiría siendo coherente pensar que el Aquinate la denomina "espiritual" porque se da en seres que poseen espíritu. Sin embargo, si seguimos adelante con el análisis notamos que no es necesaria la participación del alma para que se produzca este tipo de inmutación. En efecto, cuando en la Suma Teológica Tomás trata sobre la naturaleza de la luz afirma que el color tiene en el medio transmisor un ser intencional, el cual tiene como característica no producir cambios naturales:

Algunos dijeron que la luz en el aire no tiene ser natural, como lo tiene el color en la pared; sino ser intencional, como la semejanza del color en el aire. Pero esto no puede ser así por dos razones. La primera, porque la luz adjetiviza al aire, pues lo convierte en luminoso; pero el color no, pues no decimos aire coloreado. La segunda, porque la luz tiene su efecto en la naturaleza; por los rayos del sol se calientan los cuerpos. Por su parte, las intenciones no provocan cambios naturales. ${ }^{17}$

Además, en su Comentario al De anima da una definición de la immutatio spiritualis al decir que es "lo que sucede cuando la semejanza de un objeto es recibido en el órgano sensorio o en el medio entre el objeto y el órgano", ${ }^{18}$ demostrando que él creía que tal cambio se daba por igual en el medio y en el órgano sensorio y, por tanto, que ambos son receptivos de

15 Tomás de Aquino, S.Th. I, q.78, a.3, co.: "Spiritualis autem, secundum quod forma immutantis recipitur in immutato secundum esse spirituale; ut forma coloris in pupilla, quae non fit per hoc colorata."

${ }^{16}$ Cfr. Tomás de Aquino, In De Anima II, lectio 24, 555.

${ }^{17}$ Cfr. Tomás de Aquino, S.Th. I q. 67, a. 3, co.: "Respondeo dicendum quod quidam dixerunt quod lumen in aere non habet esse naturale, sicut color in pariete; sed esse intentionale, sicut similitudo coloris in aere. Sed hoc non potest esse, propter duo. Primo quidem, quia lumen denominat aerem, fit enim aer luminosus in actu. Color vero non denominat ipsum, non enim dicitur aer coloratus. Secundo, quia lumen habet effectum in natura, quia per radios solis calefiunt corpora. Intentiones autem non causant transmutationes naturales."

18 Tomás de Aquino, In De Anima II lectio, 14, 418: "Immutatio vero spiritualis est secundum quod species recipitur in organo sensus aut in medio per modum intentionis, et non per modum naturalis formae." 
formas inmateriales. ${ }^{19}$ A partir de estas consideraciones se observa que la inmutación espiritual puede darse en seres completamente físicos, lo que descarta la suposición de que tal cambio requiere que el objeto inmutado posea un alma.

Debemos descartar la idea de que la inmutación espiritual es un cambio mental o del alma, puesto que el mismo Tomás de Aquino afirma que la pupila y el aire son inmutados espiritualmente. Con esta evidencia textual Cohen concluye que, en el conocimiento sensible, la recepción de una forma sensible que tiene un esse intentionale (la inmutación espiritual) es un evento físico y no un evento mental, puesto que se dan en entes físicos. ${ }^{20}$

Robert Pasnau acepta la interpretación de Cohen y concluye que Tomás de Aquino es materialista con respecto a su teoría del conocimiento sensible. En efecto, si tanto la inmutación espiritual como la inmutación natural son eventos físicos, se sigue que el conocimiento sensible es un evento físico $\mathrm{y}$, por tanto, que entes absolutamente materiales, como las computadoras, son capaces de poseer tal conocimiento. ${ }^{21}$ Este comentador denomina "semimaterialista” a quien sostienen esta teoría del conocimiento.

Un semimaterialista es alguien que cree que la cognición es posible en cosas materiales. El término marca lo que me parece una distinción importante en las teorías de la mente y la cognición. Por un lado, uno puede rechazar el materialismo en el caso de los humanos y además sostener que cosas no materiales pueden ser cognoscitivas. $\mathrm{O}$ uno puede rechazar el materialismo en el caso de los humanos pero pensar que algunas cosas materiales pueden ser (o son) cognoscitivas. ${ }^{22}$

Para Pasnau los animales son entes puramente materiales capaces de tener un conocimiento sensible y, por eso, no ve ningún impedimento para afirmar que también las computadoras poseen algún tipo de conocimiento. Esto únicamente es posible si Tomás de Aquino sostuviera una posición materialista. Pero como es evidente que tal materialismo está ausente en el conocimiento humano, no es posible que el Santo sea materialista, sino que es un semimaterialista, esto es, alguien que afirma el materialismo en algunos tipos de conocimiento y pero lo niega en otros.

${ }^{19}$ Tomás de Aquino también trata sobre la forma intencional en el medio en las siguientes obras: In. Sent, lib. 2, d. 13, q. 1, a. 3 co.; In De Sensu I, 1. 5, n. 4; In De Anima, a. 4 arg. 5; De Potentia q.5, a. 8, co.; Quaestiones Quodlibetales VII, q. 1, a. 2, arg. 5.

${ }^{20}$ Cfr. Cohen S. op. cit., p. 205.

${ }^{21}$ Cfr. Pasnau R. op. cit., p. 55.

${ }^{22}$ Ibid, p. 36. 


\section{Jörg Tellkamp sobre la contradicción de la teoría del conocimiento sen- sible en Tomás de Aquino}

Teniendo en cuenta la investigación realizada por Cohen, Jörg Tellkamp estudia la inmutación espiritual tal y como la usa Tomás de Aquino en su teoría del conocimiento sensible y concluye que la teoría del Aquinate es inconsistente. La inconsistencia viene dada por el hecho de que el Santo afirma que la immutatio spiritualis es inmaterial y material al mismo tiempo. Para este autor tal contradicción puede ser observada en el estudio del concepto spiritus que maneja Tomás en ese ámbito. El Aquinate trata sobre este concepto en la Suma Contra Gentiles y afirma lo siguiente:

El nombre de espíritu parece tomado de la respiración animal, en la cual el aire es aspirado y espirado con cierto movimiento. Por eso el nombre de espíritu se aplica a todo impulso o movimiento de cualquier cuerpo aéreo, y así el viento se llama espíritu según el dicho del salmo: El fuego, el granizo, la nieve, la niebla y el viento tempestuoso, que ejecutan sus mandatos. Y se llama también espíritu al vapor sutil difundido por los miembros de los animales para sus movimientos. Igualmente, por ser invisible el aire, se ha trasladado posteriormente el nombre espíritu a todas las fuerzas y substancias invisibles y motoras. Y, por esto, el alma sensible y la racional, los ángeles y Dios, se dicen espíritus; y propiamente Dios al proceder por modo de amor, porque da a entender cierta virtud motiva. ${ }^{23}$

Como el mismo Tomás de Aquino afirma, el nombre "espíritu" es tomado primeramente de algo material que posee movimiento, la respiración animal, y luego es utilizado para hablar de las sustancias inmateriales motoras, como el alma, los ángeles y Dios. Se observa entonces que el significado primario del término spiritus tiene su origen en la teoría estoica del pneuma, utilizada posteriormente en la tradición médica, que hace referencia a un soplo puramente material. Por otra parte, este nombre también es utilizado por Tomás en las cuestiones teológicas para hablar de entes puramente inmateriales, pero con un significado diferente. Tellkamp reconoce estos dos significados opuestos que carga el concepto "espíritu":

${ }^{23}$ Tomás de Aquino, SCG Libro 4, cap, 23: "Nomen spiritus a respiratione animalium sumptum videtur, in qua aer cum quodam motu infertur et emittitur. Unde nomen spiritus ad omnem impulsum et motum vel cuiuscumque aerei corporis trahitur: et sic ventus dicitur spiritus, secundum illud Psalmi, ignis, grando, nix, glacies, spiritus procellarum, quae faciunt verbum eius. Sic etiam vapor tenuis diffusus per membra ad eorum motus, spiritus vocatur. Rursus, quia aer invisibilis est, translatum est ulterius spiritus nomen ad omnes virtutes et substantias invisibiles et motivas. Et propter hoc et anima sensibilis, et anima rationalis, et Angeli, et Deus, spiritus dicuntur: et proprie Deus per modum amoris procedens, quia amor virtutem quandam motivam insinuat." 
Hasta este punto la investigación arroja el siguiente resultado: spiritus tiene en Tomas de Aquino tres significados, de los cuales ya se mencionaron dos. (1) Uno que meramente describe el halito material de procesos corporales, (2) otro que hace alusión a la característica inmaterial sobre todo de los ángeles y de Dios y, por último, (3) la espiritualidad, concepto opuesto a lo que se refiere a lo carnal, asunto que no nos interesara aquí. ${ }^{24}$

El problema surge cuando Tomás habla de una inmutación espiritual en el contexto del conocimiento sensible y no especifica a cuál de estos significados está haciendo referencia. Si se refiere a su significado original, entonces hay que entender que la inmutación espiritual es un evento físico, tal y como lo afirma Cohen, pero en ese caso sería difícil distinguirlo de la inmutación natural. Además, el Aquinate constantemente afirma que el sentido recibe la forma sin materia, lo que obligaría a decir que la inmutación espiritual es inmaterial, concordando con el segundo significado del termino spiritus. Pero en este último caso, no se entendería por qué algunas veces denomina a la inmutación espiritual como immutatio corporalis. ${ }^{25}$ Frente a estos problemas Tellkamp concluye lo siguiente:

En la teoría del conocimiento, sobre todo en la descripción de la causalidad de procesos perceptivos, yo he tratado de defender la hipótesis que Tomas de Aquino de manera voluntaria o involuntaria, estableció una teoría con problemas de coherencia interna. Los problemas se deben sobre todo a la ambigüedad que se da entre una acepción material de spiritus, como la que acabo de describir, y una acepción inmaterial la que Tomas recogió de la tradición bíblica y del neoplatonismo. ${ }^{26}$

La lectura que realiza Tellkamp parece arrojar luz a lo dicho en el capítulo anterior. En efecto, tanto Hamlyn como Burnyeat poseían pruebas suficientes para pensar que la inmutación espiritual es un evento mental porque es una recepción inmaterial de la forma sensible. Así como la inmutación natural supone una modificación material, la inmutación espiritual supone una modificación espiritual o inmaterial. Para que haya conocimiento sensible se necesita que tanto el cuerpo como el alma sean afectados por las cualidades de los objetos externos, esto estaba asegurado por las dos inmutaciones, la natural y la espiritual.

Por otra parte, también Cohen y Pasnau poseen muchas pruebas para decir que la inmutación espiritual es un evento físico. En efecto, es imposible negar que el aire y el agua son inmutados espiritualmente porque esto es afirmado por Tomás de Aquino de manera explícita en muchas de sus

${ }^{24}$ Tellkamp J. op. cit., p. 194.

${ }^{25}$ Cfr. Tomás de Aquino, $S$. Th. I, q.75, a.3, co.

${ }^{26}$ Tellkamp J. op. cit., p. 188. 
obras. Pero también es imposible decir que tal inmutación afecta al espíritu o a la mente de estos elementos. Por eso, estos autores concluyen que la inmutación espiritual es un evento físico que puede afectar a entes completamente físicos.

Lo que nos dice Tellkamp es que todos estos autores están en lo correcto, Tomás de Aquino afirma que la inmutación espiritual es inmaterial pero también afirma que es material. Esta contradicción se debe a que no pudo sintetizar de manera adecuada dos tradiciones de pensamiento opuestas sobre el concepto de spiritus: la tradición estoica y la bíblica y neoplatónica. Cuando habla de una inmutación espiritual al tratar sobre el conocimiento sensible, el Aquinate no aclara a cuál de estas tradiciones está siguiendo, justamente porque vacila entre ellas y, por eso mismo, realiza afirmaciones que resultan ser contradictorias. Por ello concluye:

Espero haber mostrado que en la obra de Tomás de Aquino los conceptos de espíritu en general y el de inmutación espiritual en especial son problemáticos. Es constante la falta de un significado único con el cual Tomás habría podido establecer una teoría de las causas de la percepción más coherente. Nunca queda claro si al hablar de un ser espiritual y su causalidad, la inmutación espiritual, se trata de algo material o inmaterial. ${ }^{27}$

En el próximo capítulo intentaremos resolver este aparente problema siguiendo la misma línea de Tellkamp, esto es, analizando en concepto spiritus para comprender en profundidad la noción de inmutación espiritual y con ella la teoría del conocimiento sensible de Tomás de Aquino.

\section{El estado intermedio del ser sensible: el concepto de spiritus}

Pienso que el problema que señala Tellkamp se debe a que lee los textos a partir de una postura post-cartesiana, esto es, para él las cosas son materiales o inmateriales y así debe haber sido para Tomás de Aquino; pero no creo que esto sea así. En el capítulo 68 de la Suma Contra Gentiles el Aquinate habla de una jerarquía de los entes y afirma que la dignidad de un ser se debe a la manera en que la forma se desprende de la materia, mostrando que existen gradaciones en el paso de la materialidad a la inmaterialidad.

Antes bien, cuanto más noble es la forma tanto más sobrepasa en su ser a la materia. Como puede verse fijándose en las operaciones de las formas, por cuya consideración conocemos las naturalezas de las mismas, porque cada cual obra en conformidad con su ser.. ${ }^{28}$

${ }^{27}$ Ibid, p. 208.

${ }^{28}$ Tomás de Aquino, SCG, libro II, cap. 68: "Immo quanto forma est nobilior, tanto in suo esse superexcedit materiam. Quod patet inspicienti operationes formarum, ex quarum 
En ese pasaje Tomás va enumerando las formas de todos los entes compuestos de materia y forma (elementos, cuerpos mixtos, plantas, animales y hombres) y la manera en que la operación de la forma sobrepasa, o no, las cualidades dispositivas de la materia (lo cálido, lo frío, lo seco y lo húmedo). Menciona que las formas de los animales brutos están en un estado intermedio en esa escala puesto que de cierta manera sobrepasan la materia en su operación, pero en cierta medida no, ya que "sentir e imaginar no se realizan calentando o refrigerando, aunque ambas cosas sean necesarias para la buena disposición del órgano". ${ }^{29}$ Más aun, en el Comentario al De Anima, el Aquinate sostiene explícitamente que el ser sensible se encuentra en un estado intermedio entre la materialidad y la inmaterialidad:

Pero el ser inmaterial tiene dos grados en el mundo inferior. Hay una inmaterialidad perfecta del ser inteligible; las cosas existen en el intelecto no sólo sin materia, sino también sin las condiciones individuantes de la materia, y también libres de cualquier órgano material. El ser sensible está en un estado intermedio. Las cosas existen en el sentido sin materia pero no sin las condiciones individuantes de la materia, ni libres de órgano corporal. ${ }^{30}$

Lo mismo repite en el capitulo trece de las Cuestiones Disputadas sobre el alma, donde afirma que la existencia inmaterial tienen diferentes grados, mencionando nuevamente la existencia de las formas en el intelecto y en el sentido:

Por otra parte, debe haber diferentes grados de tal existencia inmaterial. Hay un grado en la medida que las cosas existen en el alma sin su materia propia, pero con su singularidad y las condiciones individuantes que se siguen de la materia. Este es el grado del sentido, que es receptivo de especies individuales sin materia, pero las recibe en un órgano corporal. El otro, que es el grado más perfecto de inmaterialidad es el del intelecto, que recibe todas las especies sin materia y abstraídas las condiciones de la material, y sin un órgano corporal. ${ }^{31}$

consideratione earum naturas cognoscimus: unumquodque enim operatur secundum quod est."

${ }^{29}$ Ibid.

${ }^{30}$ Cfr. Tomás de Aquino, In De Anima II lectio 5, 282-284: "Huiusmodi autem immateriale esse, habet duos gradus in istis inferioribus. Nam quoddam est penitus immateriale, scilicet esse intelligibile. In intellectu enim res habent esse, et sine materia, et sine conditionibus materialibus individuantibus, et etiam absque organo corporali. Esse autem sensibile est medium inter utrumque. Nam in sensu res habet esse sine materia, non tamen absque conditionibus materialibus individuantibus, neque absque organo corporali."

${ }^{31}$ Tomás de Aquino, Q. d. de anima, a. 13 co.: "Oportet autem esse diversum gradum huiusmodi esse immaterialis. Unus enim gradus est secundum quod in anima sunt res sine propriis materiis, sed tamen secundum singularitatem et conditiones individuales, quae consequuntur materiam. Et iste est gradus sensus, qui est susceptivus specierum individualium sine materia, sed tamen in organo corporali. Altior autem et perfectissimus 
Estos pasajes muestran que no todo es o bien material, o bien inmaterial, sino que existen seres que poseen en estado intermedio entre estos extremos, como la forma sensible. Como se dijo anteriormente, esta gradación se debe a que existen diferentes categorías o jerarquías en las formas y cuanto más nobles sean las formas, sus acciones más sobrepasarán las cualidades dispositivas de la materia. ${ }^{32}$

Si se tiene en cuenta los entes compuestos de materia y forma, se observa que la operación propia de los animales, esto es, el conocimiento sensible, sobrepasa a la materia pero no a la manera en que lo hace el intelecto, ya que depende de alguna inmutación material para disponer bien los órganos sensorios. Si el acto perceptivo está entre la inmaterialidad del acto intelectual y la materialidad de las operaciones del alma vegetativa, queda claro que la forma sensible también estará en un estado intermedio entre la materialidad y la inmaterialidad. Como estas formas tienen una existencia intencional en los sentidos, se concluye que la existencia intencional posee un ser semi-inmaterial en la percepción. ${ }^{33}$

Ahora bien, el término spiritus parece ser el más adecuado para representar este estado intermedio, ya que se aplica a seres tanto corporales como a los incorpóreos, como lo dice el mismo Tomás de Aquino en el Comentario a las Sentencias cuando trata sobre la definición del término spiritus, y afirma que se dice tanto de las cosas corporales como de las incorpóreas:

Respondo diciendo que "espíritu” es un nombre impuesto para significar la sutileza de alguna naturaleza. Por tanto, se dice de lo corporal tanto como de las cosas incorporales: el aire es llamado "espíritu" a causa de su sutileza, de donde se toma la atracción y expulsión del aire es llama "inspiración” y "respiración”, y el viento, también es llamado "espíritu”, y también los vapores más sutiles a través de los cuales los poderes del alma son difundidos a las partes del cuerpo son llamado "espíritu" y de la misma manera, las cosas incorpóreas son llamados "espíritus" a causa de su sutileza, incluso como nosotros llamamos a Dios, y al ángel y al alma "espíritu” (...) Ahora bien, sutileza se dice por la remoción de la materialidad. Así, esas cosas que tienen mucho de materia, como la tierra, son llamadas "pesadas", y esas que tienen menos, como el aire y el fuego son llamadas "sutiles". ${ }^{34}$

immaterialitatis gradus est intellectus, qui recipit species omnino a materia et conditionibus materiae abstractas, et absque organo corporali.” (la traducción es mía)

${ }^{32}$ Cfr. Tomás de Aquino, S. Th. I, q. 76, a. 1, co.

${ }^{33}$ Para un analisis más detallado de los grados de inmaterialidad en Tomás de Aquino Cfr. Hoffman, P. "St. Thomas Aquinas on the Halfway State of Sensible Being”, Philosophical Review 99 (1990), 73-92 y Tweedale, M. "Origins of the Medieval Theory That Sensation Is an Immaterial Reception of a Form” Philosophical Topics 20 (1992), 215-231.

34 Tomás de Aquino, In Sent lib I, dist. 10, q.1, a. 4, co.: "Respondeo dicendum, quod spiritus est nomen positum ad significandum subtilitatem alicujus naturae; unde dicitur tam 
La gradación que habíamos mencionado también se aplica a los diferentes tipos de elementos. Como el Aquinate afirma en el pasaje citado, el aire posee menos materia que la tierra, lo que lo hace más sutil. Debido a esta sutileza del aire es que se lo denomina «espiritual». Queda claro que el término espíritu puede aplicarse a seres compuesto de materia y forma, dentro de los cuales serán más espirituales aquellos que más se alejen de la materialidad. La escala de los seres desde la materialidad a la inmaterialidad coincide con la escala de la espiritualidad.

Ya habíamos mostrado que para Tomás de Aquino existen grados de inmaterialidad y que la forma sensible posee un estado intermedio entre la pura materialidad y la pura inmaterialidad. Según lo último dicho, también existen grados de espiritualidad, incluso dentro de los seres que no poseen ningún grado de inmaterialidad, en el sentido en que la operación de su forma no sobrepasa la materia. El Aquinate hace referencia a estos grados de espiritualidad al tratar sobre el conocimiento sensible y afirma que en el sentido la forma es más espiritual que en la realidad sensible.

Como la especie tiene en la realidad sensible un ser máximamente material y, en cambio, en el intelecto, sumamente espiritual, es preciso que pase a esta espiritualidad mediante ciertos grados. Así, en el sentido tiene un ser más espiritual que en la realidad sensible, y en la imaginación, todavía más espiritual que en el sentido, y de este modo va ascendiendo progresivamente..$^{35}$

Vemos que si bien el concepto spiritus no es sinónimo de inmaterial, tiene una estrecha relación con ese término porque las sustancias inmateriales son más espirituales que las que poseen materia y forma, y a su vez entre estas últimas son más espirituales aquellas donde la forma se desprende más de la materia. Parece entonces que la ambigüedad que mantiene Tomás con respecto a la inmaterialidad o materialidad de la inmutación espiritual es intencional o, a lo sumo, no resulta inconsistente con la ontología que se manejaban en esa época:

de corporalibus quam de incorporeis: aer enim spiritus dicitur propter subtilitatem; et exinde attractio aeris et expulsio dicitur inspiratio et respiratio; et exinde ventus etiam dicitur spiritus; et exinde etiam subtilissimi vapores, per quos diffunduntur virtutes animae in partes corporis, dicuntur spiritus; et similiter incorporea propter suam subtilitatem dicuntur spiritus; sicut dicimus spiritum Deum, et Angelum, et animam (...) Subtilitas autem dicitur per remotionem a materialitate; unde ea quae habent multum de materia vocamus grossa, sicut terram; et ea quae minus, subtilia, sicut aerem et ignem."

35 Tomás de Aquino, De Veritate q. 19: "Species autem in ipsa re sensibili habet esse maxime materiale, in intellectu autem summe spirituale; unde oportet quod in hanc spiritualitatem transeat mediantibus quibusdam gradibus, utpote quod in sensu habet spiritualius esse quam in re sensibili, in imaginatione autem adhuc spiritualius quam in sensu, et sic deinceps ascendendo.” 
Expresiones tales como "cuerpo sutil y ligero", "cuerpo lucido y etéreo", "vapor sutil" y "corpúsculo tenue y espiritual" (corpusculum tenue et spirituale) referían a la doble afinidad de spiritus tanto con las sustancias corporales como incorpóreas, el vínculo, pero también las confusiones y contaminaciones entre "natural y físico" y "espíritus incorpóreos" tales como el alma. Así los espíritus o cuerpos sutiles funcionaban como intermediarios y agentes entre dos mundos opuestos cuyas fronteras continuamente desplazaban y cambiaban constantemente. ${ }^{36}$

\section{Conclusión}

La dificultad que encuentran los lectores de las obras de Tomás al momento de estudiar su teoría del conocimiento sensible reside en entender cómo funciona un acto, el de percibir, que no es enteramente material ni enteramente inmaterial. La ontología que se manejaba en el tiempo de Tomás de Aquino es muy diferente a la nuestra y si perdemos de vista este hecho caeremos en errores al intentar comprender su pensamiento. Para el Aquinate no solo existen seres materiales e inmateriales, sino que también hay seres que son más materiales o más inmateriales que otros. Los animales, cuya función propia es la percepción, poseen el grado más bajo de inmaterialidad y, por eso, el ser sensible se encuentra en un estado de semiinmaterialidad.

$\mathrm{Al}$ observar que la inmutación espiritual es un proceso semi-inmaterial que se da en cosas corporales podemos ver que el mejor concepto que denota este estado intermedio es el de spiritus. A fin de cuentas, es incorrecto preguntarnos si tal inmutación es material o inmaterial, como hacen los autores que estudiamos, porque no cae bajo ninguno de estos dos rótulos. La immutatio spiritualis es una inmutación que ocurre en entes materiales pero sin producir modificaciones físicas. En la visión, por ejemplo, se puede percibir la imagen del objeto que se forma en la retina del ojo, pero no puede decirse que dicha imagen modifica materialmente al ojo, tampoco se puede afirmar que el ojo no padeció ninguna modificación porque no es lo mismo que esté esa imagen a que no esté presente en la retina.

${ }^{36}$ Neuber W. y Göttler C. Spirits Unseen: The Representation of Subtle Bodies in Early Modern European Culture, Leiden, Brill, 2008, prefacio: "Expressions such as 'thin and subtle body', 'lucid and ethereal body', 'subtle vapour' and 'fine and spiritual corpuscle' (corpusculum tenue et spiritale) referenced the double affinities of spiritus with both corporeal and incorporeal substances, the link, but also the confusions and contaminations between 'physical and natural' and 'incorporeal spirits' such as the soul. Spirits or subtle bodies thus functioned as intermediaries and agents between two opposite worlds whose borders continually shifted and changed." 
Podemos pensar que Tomás de Aquino era consciente de la ambigüedad del significado que portaba el concepto de spiritus y, por eso mismo, decide utilizarlo para describir a la inmutación que padece el sentido. Tal inmutación no puede ser material, ya que se confundiría con la inmutación natural, pero tampoco puede ser una inmutación de la mente, puesto que la forma sensible es recibida en los órganos sensorios, que son materiales, incluso debe ser transportada por un medio material para que pueda percibirla el ente cognoscitivo.

\section{Bibliografía}

Aristóteles, Acerca del alma, introducción, traducción y notas de Tomás Calvo, Madrid, Gredos, 1978.

Burnyeat, M. "Is an Aristotelian Philosophy of Mind Still Credible? (A Draft)", Essays on Aristotle's de Anima (1995), 15-26.

Cohen, S. "St. Thomas Aquinas on the Immaterial Reception of Sensible Forms", Philosophical Review 91 (1982), 193-209.

Hamlyn, D. Sensation and Perception, London, Routledge \& Kegan Paul, 1961.

Hoffman, P. "St. Thomas Aquinas on the Halfway State of Sensible Being”, Philosophical Review 99 (1990), 73-92.

Neuber, W. y Göttler, C. Spirits Unseen: The Representation of Subtle Bodies in Early Modern European Culture, Leiden, Brill, 2008.

Pasnau, R. Theories of Cognition in the Later Middle Ages, Cambridge, Cambridge University Press, 1997.

Tellkamp, J. "Espíritu e inmutación espiritual: desarrollos y problemas en Tomás de Aquino", Tópicos: Revista de Filosofía 15 (1998), 181-210.

Tomás de Aquino, Comentario al libro de Aristóteles sobre el sentido y lo sensible, traducción de Juan Cruz Cruz. Pamplona, EUNSA, 2001.

Tomás de Aquino, Commentary on Aristotle's De Anima, traducción de Kenelm Foster, O.P. y Sylvester Humphries, O.P. New Haven, Yale University Press, 1951

Tomás de Aquino, Compendio de Teología, traducción y notas de J. I. Saranyana y J. Restrepo, Madrid, Ed. Rialp S. A., 1980

Tomás de Aquino, De Potentia Dei 5, La conservación, introducción, traducción y notas de Nicolás Prieto, Pamplona, Servicio de Publicaciones de la Universidad de Navarra, 2005.

Tomás de Aquino, De Veritate, Cuestión 19: sobre el conocimiento del alma tras la muerte, introducción, traducción y notas de José Ignacio Murillo, 
Pamplona, Servicio de Publicaciones de la Universidad de Navarra, 1999. Tomás de Aquino, Quaestiones Disputatae de anima, traducido como On Soul por John Patrick Rowan, St. Louis \& London, B. Herder Book Co, 1949.

Tomás de Aquino, Quaestiones de Quodlibet. Quodlibet VII, VIII, IX, X, XI, Roma-París, Commisio Leonina-Èditions du Cerf, 1996.

Tomás de Aquino, Opera omnia, t. 7/2: Commentum in quartum librum Sententiarum magistri Petri Lombardi, Parma, Typis Petri Fiaccadori, 1856.

Tomás de Aquino, Suma Contra los Gentiles, Madrid, Editorial Católica, 1952.

Tomás de Aquino, Suma Teológica, vol. 1, Madrid, Biblioteca de Autores Cristianos, 1947.

Tomás de Aquino, Suma Teológica, vol. 4, Madrid, Biblioteca de Autores Cristianos. 1947.

Tomás de Aquino, Suma Teológica, vol. 13, Madrid, Biblioteca de Autores Cristianos, 1957.

Tweedale, M. "Origins of the Medieval Theory That Sensation Is an Immaterial Reception of a Form" Philosophical Topics 20 (1992), 215-231. 